ISSN 1014-4874

DOI : http://dx.doi.org/10.4314/rj.v27i1.6

\title{
Deploying Affordable and Available Telecommunications Infrastructure for the Education Sector in The Gambia
}

Dembo Ibrahim Sankareh, Dr. Adronis Niyonkuru, Dr. Felix K. Akorli

Corresponding author : demboibrahim@gmail.com

\begin{abstract}
This paper focuses on key challenges impeding the deployment of Internet Connectivity to Schools, implementation of ICT in education and provision of requisite ICT tools to enhance delivery and quality of education in The Gambia. Given the fact that implementation of submarine cable and landing station for The Gambia(Africa Coast to Europe -ACE) is at advanced stage and implementation of National Fibre Optic Backbone infrastructure is ongoing, leveraging these two major telecommunications projects to address the telecommunications needs of educational institutions is also looked into.
\end{abstract}

\section{Introduction}

Since the advent of Internet to The Gambia, The Gambia has been connected to the SAT-3 submarine cable with two STM1s for voice and data communications. With the passage of time and unfolding of developments in the telecommunications market, price for international bandwidth had increased several times and bandwidth for SAT-3 submarine cable exhausted. This coupled with lack of complete national fibre optic backbone infrastructure hinders expanding availability of telecommunications services to other sectors and areas hence widening the digital divide. In this respect, the education sector, which is the focus of this paper, is not the least affected. The participation of The Gambia to ACE project [1] and the expansion and upgrading of the national telecommunications backbone infrastructure to an ALL-IP network will address the inherent connectivity gaps, substantially increase international bandwidth, foster regional integration and enhance economic and Small Medium Enterprises (SME) growth. 


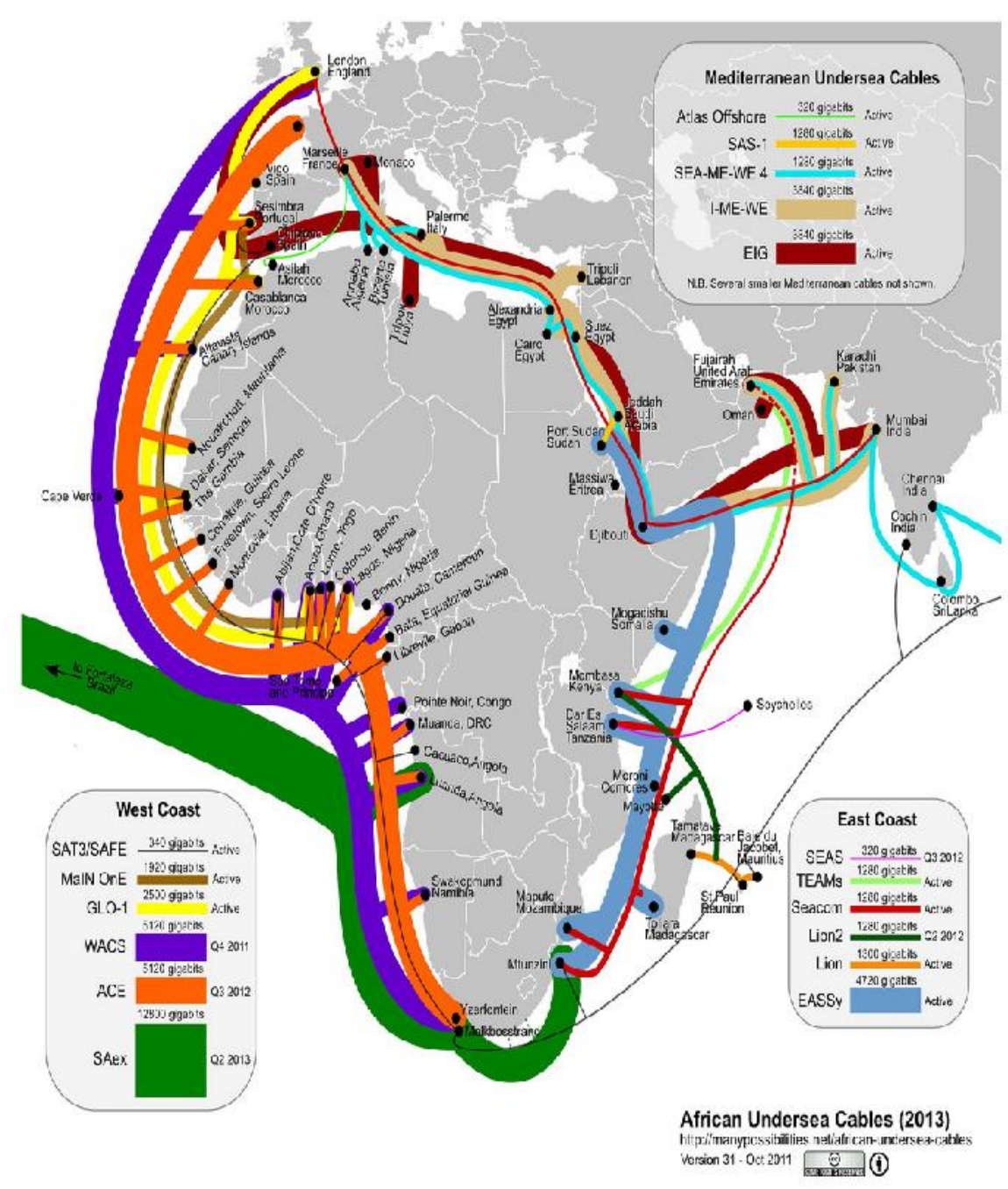

Figure 1: Submarine Cable routes in Africa.

\section{Current Status}

In this era of Information and Communications Technologies (ICTs), the importance of a modern telecommunications infrastructure cannot be overemphasized. A modern telecommunications infrastructure provides access which is a cornerstone for bridging the digital divide. Along this line and as part of the Worldlinks Programme funded by the World Bank, the Ministry of Basic and Secondary Education of The Gambia from 2005-2006 implemented 20 ICT laboratories for 20 Senior Secondary Schools. These laboratories are equipped with networked computers, Internet access and 
other computer peripherals. Hitherto, implementation of ICT laboratories for Senior Secondary Schools started in 2000.

The education sector is faced with myriad of challenges with regard to adoption of ICT to enhance teaching and learning. The main challenges are:

1. Infrastructure: Expensive internet connectivity to Schools ICT laboratories continue to pose challenges for sustaining existing laboratories and rolling out more. Most Schools in Urban areas have ICT laboratories with internet connectivity. As for the rural areas, very few are connected to the internet due mainly to lack of coverage by the Internet Service Providers (ISPs), area not covered by the Rural Electrification Project (REP). This creates UrbanRural digital divide.

2. ICT in Education: A greater percentage of private schools have included ICT in their curriculum [2]. ICT curriculum has not been developed yet for Government and grant-aided schools. Lack of qualified ICT teachers is also another factor hindering the teaching and learning through ICT in public schools.

3. Resources: Low Computer to Student ratio is another factor that should be addressed. Usually, there are less than 50 computers in schools ICT laboratories to be used by an entire School population on turn basis. Given the constraint in providing computers and other peripherals to educational institutions, the Education Ministry should join the One Laptop Per Child (OLPC) program and explore possibilities of implementing the program in The Gambia.

\section{Proposed Solution}

The above section have given detail analysis of the challenges the Education sector is faced with in deploying requisite and sustainable telecommunications infrastructure, usage and adoption of ICT in teaching and learning not only to enhance quality and delivery of education but to prepare students harness full potentials of ICT thus contributing effectively and participating confidently in the global information society and knowledge economy.

In light of the above, it is evident that, affordable and available telecommunications and internet infrastructure which will provide connectivity inter alia to all schools and research institutions to facilitate learning and research is needed. Accordingly, The Gambia Research and Education Network is hereby proposed. The proposed infrastructure of The Gambia NREN is shown Figure 2. 


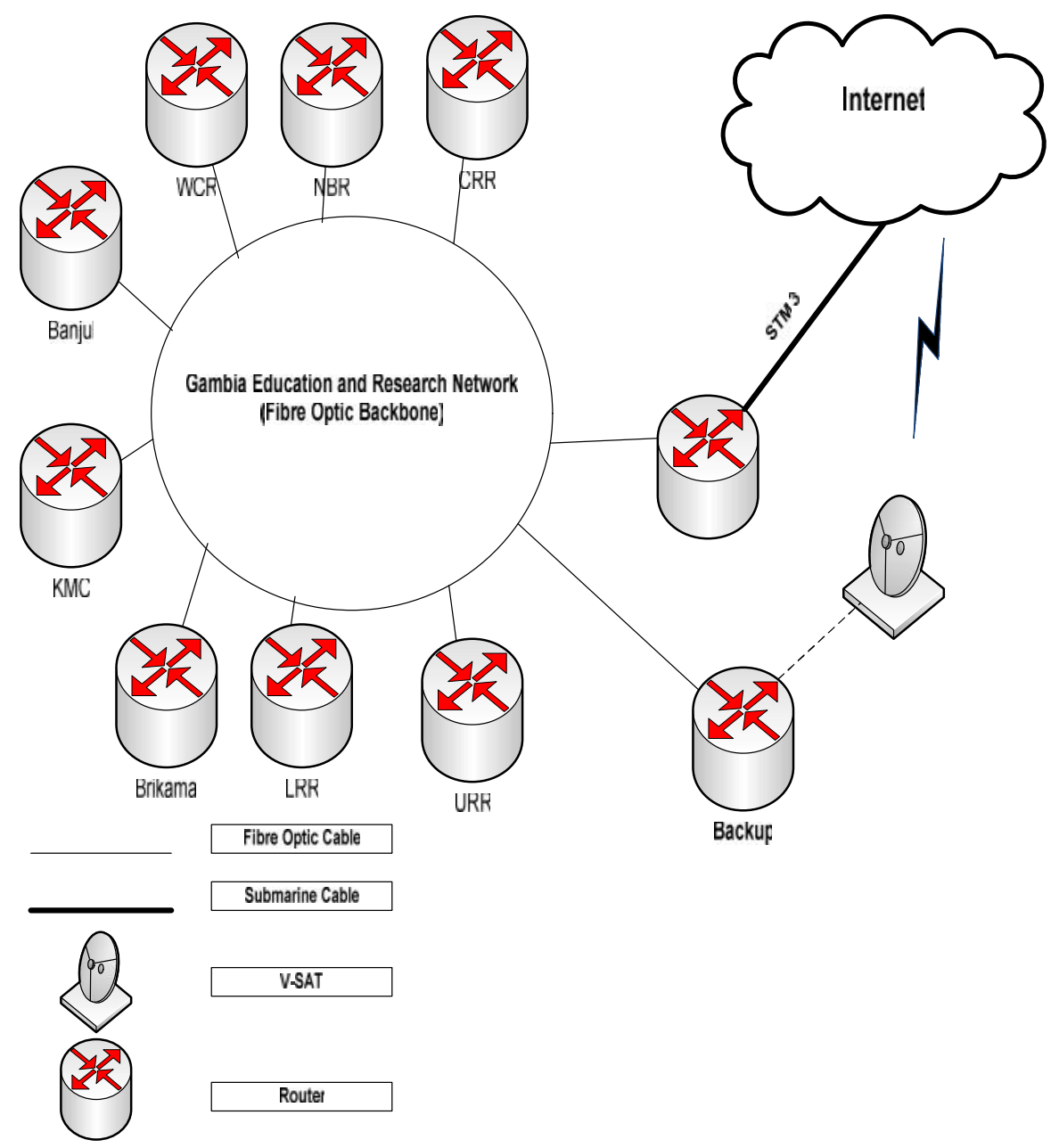

Figure 2: Proposed Infrastructure

The proposed Gambia NREN structure is made up of three (3) layers:

1. National Fibre Optic Backbone Network: This is a dark fibre optic backbone network. All regional network centres are linked to this core network. The core network in turn link regional centres to the Internet using STM3.

2. Regional Networks: The main regions in The Gambia are: Banjul, KMC, Brikama, West Coast Region (WCR), Lower River Region (LRR), North Bank Region (NBR), Central River Region (CRR) and Upper River Region (URR). Hence, the above mentioned regional networks. Regional networks serve the regions in which they are built. 
3. Campus Networks: This can refer to networks in schools of all levels, research institutes, libraries, regional education departments. Campus networks are connected to regional networks Wireless Technology for last mile connectivity.

The participation of The Gambia Government to the ACE project and implementation of the National Telecommunications backbone infrastructure are important ingredients towards the implementation of the proposed NREN for the Gambia. As for the ACE project -The Gambia component, there are provisions for Government to allocate STM3 for social services (e.g. Education sector, Health, etc). At any rate, it will be far cheaper compared to commercial providers should government decide to attach some charges for allocation of STM3 for social services. With regard to the national fibre optic backbone, which will be implemented and managed by government owned telecommunications company, GAMTEL, it is more cost effective for government to grant Indefeasible Rights of Use (IRU) of the dark fibre to the proposed NREN [3]. Cognizant of the fact that NREN's are evolving to be responsive to the demands of the $21^{\text {st }}$ century [4], the proposed infrastructure is designed with that precondition in mind.

\section{Conclusion}

This paper is being written at a time when the ACE submarine cable has landed in The Gambia and is expected to be operational by second quarter of 2012; National fibre optic backbone infrastructure being expanded and upgraded. Recognising ICTs as a catalyst for socioeconomic development and the contribution of NRENs to knowledge economy and information society, The Gambia government will be allocating STM3 for social services. This will ease implementation and sustainability of the proposed Gambia NREN.

The proposed NREN will be providing myriad of services including training, technical support, network services, content creation etc.

\section{Acknowledgement}

This research has been facilitated by UNESCO under the UNESCO/Keizo Obuchi Research Fellowships Programme (UNESCO/Japan Young Researchers Fellowships Programme), Cycle 2011. The Fellowship is funded by the Government of Japan. I would like to register my appreciation and thanks to the Government of Japan for honoring its commitment to impact on capacity building by allocating financial resources to fellows like my humble self to undertake research in areas of development. 
I am indebted to colleagues at the Ministry of Information and Communications Infrastructure and Ministry of Basic and Secondary Education of the Republic of The Gambia for their support.

Special thanks to my research supervisor, Dr. Adronis Niyonkuru and Dr. Felix K. Akorli for their support.

\section{References \& Resources}

[1] ACE Consortium website www.ace-submarinecable.com

[2] African Under Sea Cables http://manypossibilities.net/african-underseacables/

[3] Kennedy Aseda. "Kenya Education Infrastructure"

[4] Ashraf Patel, 2010. "New Models for NREN's and Universities in the era of Broadband and Web 2.0 Applications and Innovations," Proceedings of and report of the $3^{\text {rd }}$ UbuntuNet Alliance annual conference 